\title{
Proper motions of radiative knots in simulations of stellar jets
}

\section{An alternative to pulsating inflow conditions}

\author{
F. Rubini ${ }^{1}$, S. Lorusso ${ }^{1, \star}$, L. Del Zanna ${ }^{1}$, and F. Bacciotti ${ }^{2}$ \\ 1 Dipartimento di Astronomia e Scienza dello Spazio, Università di Firenze, Largo E. Fermi 2, 50125 Firenze, Italy \\ e-mail: rubini@arcetri.astro.it \\ 2 INAF - Osservatorio Astrofisico di Arcetri, Largo E. Fermi 5, 50125 Firenze, Italy
}

Received 13 April 2007 / Accepted 20 June 2007

\begin{abstract}
Aims. Elongated jets from young stellar objects typically present a nodular structure, formed by a chain of bright knots of enhanced emission with individual proper motions. Though it is generally accepted that internal shocks play an important role in the formation and dynamics of such structures, their precise origin and the mechanisms behind the observed proper motions is still a matter of debate. Our goal is to study numerically the origin, dynamics, and emission properties of such knots.

Methods. Axisymmetric simulations are performed with a shock-capturing code for gas dynamics, allowing for molecular, atomic, and ionized hydrogen in non-equilibrium concentrations subject to ionization/recombination processes. Radiative losses in [S II] lines are computed, and the resulting synthetic emission maps are compared with observations.

Results. We show that a pattern of regularly spaced internal oblique shocks, characterized by individual proper motions, is generated by the pressure gradient between the propagating jet and the time variable external cocoon. In the case of under-expanded, light jets the resulting emission knots are found to move downstream with the jet flow, with increasing velocity and decaying brightness toward the leading bow shock. This suggests that the basic properties of the knots observed in stellar jets can be reproduced even without invoking ad hoc pulsating conditions at the jet inlet, though an interplay between the two scenarios is certainly possible.
\end{abstract}

Key words. ISM: Herbig-Haro objects - ISM: jets and outflows - stars: winds, outflows - hydrodynamics - shock waves methods: numerical

\section{Introduction}

Collimated outflows and jets seem to be ubiquitous features in astrophysics and are observed over a wide range of spatial scales, from several megaparsecs for extragalactic sources (AGNs) down to a few parsecs for young stellar objects (YSOs). In both cases a chain of bright knots is typically observed along the jet, suggesting that similar physical processes may be at work in spite of the enormous difference in scale and, most probably, even in the jet composition itself. As far as YSO jets are concerned, the bright knots observed in emission lines (also known as Herbig-Haro, $\mathrm{HH}$, objects) represent spectacular tracers for these structures over a wide range of wavelengths, and their ubiquity suggests that they are a key component of the jet dynamics.

Though widely observed in detailed images and spectra (e.g. Reipurth \& Bally 2001), the precise nature and origin of the knots is, however, still the subject of investigation. In the first bright section of an optical jet, up to $\approx 0.1 \mathrm{pc}$ from the source, the number of knots ranges typically between 5 and 20. Their pattern is not static, but moves at a substantial fraction, from $70 \%$ or more, of the flow speed, a value increasing with distance from the source (Eislöffel \& Mundt 1992). On the other hand, the knots brightness tends to decay along the jet axis (Morse et al. 1992; Ray et al. 1996; Reipurth \& Bally 2001). Several jets have been imaged at high resolution $\left(\$ 0.1^{\prime \prime}\right)$ using the Hubble Space Telescope (HST) (Ray et al. 1996; Reipurth et al. 1997; Hartigan et al. 2001;

^ Now at: GE Oil \& Gas, Nuovo Pignone, Firenze, Italy
Bally et al. 2002; Reipurth et al. 2002; Hartigan et al. 2005). As an example, HST images of $\mathrm{HH} 46 / 47$ exhibit a complex structure in which the [S II] emission decouples from the $\mathrm{H} \alpha$ emission (Heathcote et al. 1996). Both lines define a chain of small knots with spacing of $2-3^{\prime \prime}$. The $\mathrm{H} \alpha-$ [S II] difference image shows that the [S II] emission is more extended along the flow than in $\mathrm{H} \alpha$ emission maps, where filaments and wisps are visible, produced either at the front of internal working surfaces or in shock induced local changes in the flow direction. In this framework the $\mathrm{H} \alpha$ emission is produced in the hotter and more excited thin layer just behind the shock front, while [S II] lines come from a denser and cooler layer more distant from the front, which has a larger extension (Bacciotti \& Eislöffel 1999). HH 47 also shows a set of leading bow shocks with spacing much larger than the intervals between neighboring knots.

The current interpretation for the origin of both the beam mini bow shocks and the large leading bow shocks involves the presence of pulsation in the ejection mechanism (Raga \& Kofman 1992; Stone \& Norman 1993; Falle \& Raga 1995; Suttner et al. 1997; Raga et al. 1998, 2002) (the working surfaces scenario). The physical reason for such pulsation, however, has yet not been clearly identified. It is possible that some kind of self-consistent MHD mechanism is at work (e.g. Ouyed \& Pudritz 1997). A pulsating inflow has also been applied to 3D simulations which include the effects of precession due to the rotation of the nozzle (Cerqueira \& de Gouveia Dal Pino 2004; Cerqueira et al. 2006), motivated by the recent observations of toroidal velocities at the base of jets associated with T Tauri stars (Bacciotti et al. 2002; Coffey et al. 2004; Woitas et al. 2005; 
Coffey et al. 2007). Finally, the effects of magnetic fields in axisymmetric simulations with periodically varying inflow conditions have been also studied (Cerqueira et al. 1997; O'Sullivan \& Ray 2000; Stone \& Hardee 2000; Massaglia et al. 2005; de Colle \& Raga 2006).

On the other hand, other HH jets such as HH 30 (Ray et al. 1996; Bacciotti et al. 1999) do not present bow shock-like features in the forbidden lines, and the knots look more like axially symmetric blobs aligned with the jet axis. It has been suggested in the past that this appearance can be generated by Kelvin-Helmholtz instabilities. In particular, Bodo et al. (1994) have investigated the growth of Kelvin-Helmoltz instabilities in a Cartesian slab of supersonic, adiabatic flow interacting with the external matter at rest. In their paper the authors show that as these instabilities grow linearly and eventually saturate, a diamond-like pattern of internal oblique shocks (IOS) is formed. However, the periodic boundary conditions imposed on the longitudinal jet propagation direction prevent all effects due to the non-stationary jet propagation and necessarily yield IOS patterns which are static with respect to the mean flow.

In the present study we perform numerical simulations of cooling jets which originate from a nozzle, to recover the effects of non-stationary jet propagation, but we do not impose a periodically variable inflow velocity as it is generally assumed. Encouraged by our preliminary results (Rubini et al. 2004), where intermittent patterns of knots were found in some particular cases even without ad hoc assumptions on the inflow speed, our intention here is to study, in greater detail and for a wider choice of parameters, the formation mechanism, kinematics, and emission properties of the IOS which are seen to form in the region behind the jet head. These arise due to the pressure gradient between the jet and the external medium, and may be ultimately responsible for the observed knotty emission. 3D effects, such as those due to precession motions at the nozzle or to kink-like instabilities induced by magnetic field, are expected to be more important far away from the source. For this reason, a simple axisymmetric hydrodynamical model can be used when investigating the region near the nozzle, where knots are seen to form and appear still well collimated with the jet beam. Moreover, a cooling model based on a three-species network (neutral, ionized, and molecular hydrogen) can be safely assumed since we are not interested in resolving the small cooling scales behind the leading bow-shock, where $\sim 10-20$ species are typically evolved. For recent results on this subject see Raga et al. (2007) and references therein.

The steady inflow scenario had been investigated by other authors, but it was argued that such IOS should be smoothed away in radiative jets by the cooling losses (e.g. Blondin et al. 1990). Cerqueira et al. (1997) also remarked that in magnetized jets the cooling tends to smooth out pinch-like instabilities. In general, it is agreed that radiative losses tend to reduce the shock strength (Downes \& Ray 1998; Micono et al. 1998, 2000), so the idea has been put aside in favor of a pulsating inflow. However, by using the most recent indications concerning the physical parameters of the gas at the jet base, obtained from spectral diagnostics of high angular resolution data (e.g. Lavalley-Fouquet et al. 2000; Bacciotti 2002; Hartigan et al. 2004), it is possible to determine the initial conditions of the numerical simulations in a more realistic way than has been done in the past. Here we are able to show that IOS are able to survive the cooling losses and to form a regular pattern of emitting knots on length scales which mostly depend on the pressure ratio between jet and interstellar medium. Moreover, such a pattern is not static, but the individual knots show a degree of proper motion even when steady inflow conditions are imposed. This result is basically due to the fact that the gas surrounding the jet beam, the cocoon, is a highly dynamic and time-varying environment. Thus, the interpretation of the optical knots as being due to IOS cannot be ruled out and most probably cooperates with the other effects successfully proposed so far.

The paper is structured as follows. In Sect. 2 we discuss the equations, the numerical method, and the general mechanism for the formation of IOS and for their proper motion. In Sect. 3 we present various results from the simulations, including a comparison with observations. Section 4 is devoted to the final discussions.

\section{The physical and numerical model}

\subsection{Gas dynamic equations and source terms}

In our simulations we solve the system of hydrodynamical fluid equations for a cooling gas formed by molecular $\left(\mathrm{H}_{2}\right)$, atomic (HI), and ionized (H II) hydrogen in non-equilibrium concentrations, plus atomic helium $(\mathrm{He})$ and heavier elements in fixed concentrations and free electrons (e). The evolution equations for such a system, in conservation form as required by shockcapturing numerical schemes, are the following:

$$
\frac{\partial \rho}{\partial t}+\boldsymbol{\nabla} \cdot(\rho \boldsymbol{u})=0
$$

$\frac{\partial}{\partial t}(\rho \boldsymbol{u})+\boldsymbol{\nabla} \cdot(\rho \boldsymbol{u} \boldsymbol{u}+p \boldsymbol{I})=\mathbf{0}$

$\frac{\partial}{\partial t}(\rho E)+\boldsymbol{\nabla} \cdot(\rho H \boldsymbol{u})=-\mathcal{S}_{\mathrm{rad}}-\mathcal{S}_{\mathrm{ion}}-\mathcal{S}_{\mathrm{H}_{2}}$

$\frac{\partial N_{\mathrm{H} I I}}{\partial t}+\boldsymbol{\nabla} \cdot\left(N_{\mathrm{H} \text { II }} \boldsymbol{u}\right)=\mathcal{N}_{\mathrm{ion}}-\mathcal{N}_{\mathrm{rec}}$

$\frac{\partial N_{\mathrm{H}_{2}}}{\partial t}+\boldsymbol{\nabla} \cdot\left(N_{\mathrm{H}_{2}} \boldsymbol{u}\right)=-\mathcal{N}_{\text {diss }}$

In the above equations $\rho, \boldsymbol{u}$ and $p$ represent density, velocity and pressure for each volume element, $\boldsymbol{I}$ is the identity tensor, $E=$ $\varepsilon+\frac{1}{2} u^{2}$ is internal plus kinetic energy per unit mass, and $H=$ $E+p / \rho$ is the specific enthalpy. These evolution equations are completed by the relation for the total number density

$N_{\text {tot }}=N_{\mathrm{H} \text { II }}+N_{\mathrm{HI}}+N_{\mathrm{H}_{2}}+N_{\mathrm{e}}+N_{\mathrm{He}}$,

which is used to derive the gas temperature, where $N_{\mathrm{H} \text { II }}, N_{\mathrm{HI}}$, $N_{\mathrm{H}_{2}}, N_{\mathrm{e}}$, and $N_{\mathrm{He}}$ are the number densities for ionized, atomic (neutral), molecular hydrogen, electrons, and helium atoms, respectively. The source terms on the right hand side of Eq. (3), the energy equation, are respectively: $\mathcal{S}_{\text {rad }}$, which contains all radiative losses excepting those due to $\mathrm{H}_{2} ; \mathcal{S}_{\mathrm{H}_{2}}$, where radiative and dissociation losses from $\mathrm{H}_{2}$ are included; $\mathcal{S}_{\text {ion }}$, which takes into account hydrogen ionization. These contributions, which require computation of the particle densities, will be discussed later. Note that the non-equilibrium hypothesis leads to the two extra continuity equations, namely Eq. (4) for protons and Eq. (5) for molecular hydrogen. In the former, the term $\mathcal{N}_{\text {ion }}-\mathcal{N}_{\text {rec }}$ is the proton population variation, per unit of time and volume, due to ionization and recombination, whereas in the latter $\mathcal{N}_{\text {diss }}$ is the molecular hydrogen density decrease rate due to dissociation processes. Reformation processes have not been considered here, since densities for molecular hydrogen reformation are too small, and reformation time scales are too large compared with 
dissociation time scales. Solar abundances of heavier elements in fixed concentrations have been also included, since they are important for energy losses though are too small to affect the mass.

For the computation of the total particle density in Eq. (6), which is needed to update the temperature field, the following steps are required:

- the total mass density $\rho$ and the number densities $N_{\mathrm{e}}$ and $N_{\mathrm{H}_{2}}$ are evolved directly via the respective continuity equations, thus are known quantities at each time-step;

- let us first introduce the total number density of hydrogen atoms as

$N_{\mathrm{H}}=N_{\mathrm{H} \text { II }}+N_{\mathrm{HI}}+2 N_{\mathrm{H}_{2}}$,

including all possible states. From the relations $\rho_{\mathrm{H}}=N_{\mathrm{H}} m_{\mathrm{p}}$, $\rho_{\mathrm{He}}=4 N_{\mathrm{He}} m_{\mathrm{p}}\left(\rho=\rho_{\mathrm{H}}+\rho_{\mathrm{He}}\right.$ and $m_{\mathrm{p}}$ is the proton mass $)$, and assuming helium to be present in a fixed concentration with $\rho_{\mathrm{He}} / \rho=1 / 4$, we easily find

$N_{\mathrm{H}}=\frac{3}{4}\left(\rho / m_{\mathrm{p}}\right), \quad N_{\mathrm{He}}=\frac{1}{16}\left(\rho / m_{\mathrm{p}}\right) ;$

- the number density of electrons $N_{\mathrm{e}}$ is derived from

$N_{\mathrm{e}}=N_{\mathrm{H} \text { II }}+N_{\mathrm{em}}=N_{\mathrm{H} \text { II }}+10^{-4} N_{\mathrm{H}}$,

where $N_{\mathrm{em}}$ is the concentration of free electrons due to metals, again assumed to be a fixed fraction of $N_{\mathrm{H}}$;

- the number density of atomic hydrogen $N_{\mathrm{HI}}$ is finally derived from Eq. (7).

Once we have the temperature field, we are ready to compute all source terms in the fluid equations. Let us discuss them in detail. The source term in Eq. (4) for ionized hydrogen is provided by recombination and ionization rates, respectively given by

$\mathcal{N}_{\text {rec }}=N_{\mathrm{e}} N_{\mathrm{H} \text { II }} \alpha(T), \quad \mathcal{N}_{\text {ion }}=N_{\mathrm{e}} N_{\mathrm{H} \text { I }} \beta(T)$.

The functions $\alpha$ and $\beta$ of temperature $T$ (in Kelvin degrees) are

$\alpha(T)=2.06 \times 10^{-11} T^{-1 / 2} \phi \mathrm{cm}^{3} \mathrm{~s}^{-1}$,

$\beta(T)=7.80 \times 10^{-11} T^{1 / 2} \exp \left(-13.6 \mathrm{eV} / k_{\mathrm{B}} T\right) \mathrm{cm}^{3} \mathrm{~s}^{-1}$,

where $\phi$ in Eq. (11) is a decreasing function of the temperature, ranging approximately from 4 to 1 in the region of interest (see Table 5.2 in Spitzer 1978), whereas Eq. (12) is taken from Lang (1975) for hydrogen neutral atoms mainly in the ground level, as expected for a low density gas, and $k_{\mathrm{B}}$ is the Boltzmann constant. This yields an energy loss contribution $\mathcal{S}_{\text {ion }}=\left(\mathcal{N}_{\text {ion }}-\mathcal{N}_{\text {rec }}\right)$. $13.6 \mathrm{eV}$ due to ionization of hydrogen in Eq. (3).

The source term in the molecular hydrogen equation arises from molecular dissociation alone, due to collisions with $\mathrm{H}_{2}, \mathrm{HI}$, $\mathrm{H}$ II and electrons. This yields

$\mathcal{N}_{\text {diss }}=N_{\mathrm{H}_{2}}\left[N_{\mathrm{H}_{2}} K_{\mathrm{H}_{2}}+N_{\mathrm{HI}} K_{\mathrm{H}_{\mathrm{I}}}+N_{\mathrm{H} \text { II }} K_{\mathrm{H} \text { II }}+N_{\mathrm{e}} K_{\mathrm{e}}\right]$,

in which dissociation rates $K_{\mathrm{H}_{2}}$ and $K_{\mathrm{H}_{\mathrm{I}}}$ due to collisions with neutrals, $\mathrm{H}_{2}$ and $\mathrm{HI}$, respectively, are computed through the model by Lepp \& Shull (1983), whereas those due to collisions with charged particles, $K_{\mathrm{H} \text { II }}$ and $K_{\mathrm{e}}$, derive from Hollenbach \& McKee (1989) and Mac Low \& Shull (1986). Corrections to the critical density in order to separate high and low density regimes, as suggested by Martin et al. (1996), have also been introduced. The corresponding contribution to the total energy source is $\mathcal{S}_{\mathrm{H}_{2}}=\Lambda_{\text {rad }}+\Lambda_{\text {diss }}$, where $\Lambda_{\text {diss }}=\mathcal{N}_{\text {diss }} \cdot 4.48 \mathrm{eV}$ comes from dissociations while $\Lambda_{\text {rad }}$ is mainly due to collisions with $\mathrm{HI}$ and $\mathrm{H}_{2}$ (see Lepp \& Shull 1983).
Finally, we consider the cooling function $\mathcal{S}_{\text {rad }}$. For $T>10^{4} \mathrm{~K}$ we use the cooling function as in Dalgarno \& McCray (1972), which considers radiative losses due to de-excitation of H I energy levels. Here we assume the gas to be optically thin. For $T<10^{4} \mathrm{~K}$ the cooling function is extended by computing energy losses mainly due to collisions between electrons and $\mathrm{C}$ II, O I, N I, Fe II, O II, S II, or, for still lower temperatures, between H I and C II, OI, Si II, Fe II (Bacciotti et al. 1995). Moreover, we must consider that in star-forming regions, refractory species concentrate at the surface of dust grains. The cooling function has been corrected accordingly to take into account reduced concentrations with respect to standard metal abundances (Sofia et al. 1994).

\subsection{Simulation setup and choice of parameters}

The numerical code used in our simulations is a finite volume Godunov-type scheme which solves Eqs. (1)-(5), in 2D, by assuming axisymmetry around the jet axis and adopting cylindrical coordinates $(\xi, r)$, where $\xi$ is the distance from the source along the jet and $r$ is the radius. We use second order limited reconstruction on characteristic variables and an exact Riemann solver at cell interfaces to work out numerical fluxes, which in our simulations has turned out to be more robust with respect to a Roe solver. The jet originates from a nozzle of a given radius, located at the left side of the numerical box and then propagates in the $\xi$ direction. Convergence tests have been performed to find the optimal numerical parameters which reconcile accuracy and efficiency. A stretched grid with 150 points is used in the radial direction, which uses 20 points to describe the nozzle region, from $r=0$ to $r=r_{\text {jet }}$, whereas along $\xi$ we expand the numerical box by adding points (up to 200 at most), while maintaining a fixed resolution, as the jet propagates in the unperturbed medium. Details of the numerical method and tests may be found in Lorusso (1999).

The physical parameters to be initialized at the nozzle and in the external, unperturbed interstellar medium (ISM), are density, pressure (and, consequently, temperature), ionization and molecular hydrogen fractions (defined as $x_{\mathrm{e}} \simeq x_{\mathrm{H} \text { II }}=$ $N_{\mathrm{H} \text { II }} /\left(N_{\mathrm{HI}_{\mathrm{I}}}+N_{\mathrm{H} \text { II }}\right)$ and $x_{\mathrm{H}_{2}}=N_{\mathrm{H}_{2}} / N_{\mathrm{H}}$, respectively $)$, plus jet radius $r_{\text {jet }}$ and inflow velocity $V_{\text {jet }}$. Most of these parameters can be derived from observations, either directly or combined with spectral diagnostics at moderate and high angular resolution (Bacciotti \& Eislöffel 1999; Lavalley-Fouquet et al. 2000; Bacciotti et al. 2002). Typical observed tangential velocities are about $100-200 \mathrm{~km} \mathrm{~s}^{-1}$ and we assume $V_{\text {jet }}=200 \mathrm{~km} \mathrm{~s}^{-1}$ (which corresponds to a Mach number of the order of 20 for the assumed temperature, see below).

In general, at 100-200 AU from the source, the ionization fraction $x_{\mathrm{e}}$ and the electron density $N_{\mathrm{e}}$ in the jet are in the range $0.2-0.5$ and $10^{2}-10^{3} \mathrm{~cm}^{-3}$, respectively, while the temperature is in the range $6000-10000 \mathrm{~K}$. From this point onward the chain of knots begins to be visible and the physical quantities mentioned above oscillate around the values attained at $200 \mathrm{AU}$ from the source (see, e.g., Figs. 11 and 2 in Bacciotti et al. 1999). According to these analyses we place the inlet at about $200 \mathrm{AU}$ from the star.

The precise parameters for all runs are shown in Table 1. Rather than giving the jet density and pressure, we fixed only the ISM values and we prescribed the two quantities $\eta=\rho_{\text {jet }} / \rho_{\text {ISM }}$ and $\Pi=p_{\text {jet }} / p_{\text {ISM }}$. Namely, the jet density and pressure are derived from the given values assuming $\rho_{\mathrm{ISM}}=0.5 \bar{\rho}$ and $p_{\text {ISM }}=\bar{p}$. Normalization is against $\bar{L}=10^{15} \mathrm{~cm}, \bar{\rho}=1.673 \times$ $10^{-20} \mathrm{~g} \mathrm{~cm}^{-3}$ (corresponding to $10^{4}$ hydrogen atoms per $\mathrm{cm}^{3}$ ), 
Table 1. Run parameters. According to our definitions, $\rho_{\text {jet }}=\eta 0.836 \times$ $10^{-20} \mathrm{~g} \mathrm{~cm}^{-3}, p_{\text {jet }}=\Pi 1.381 \times 10^{-10} \mathrm{erg} \mathrm{cm}^{-3}$. The jet velocity is always assumed $V_{\text {jet }}=200 \mathrm{~km} \mathrm{~s}^{-1}$. Case $\mathcal{E}$ is the same as $\mathcal{D}$ but with restarting inflow conditions.

\begin{tabular}{cccccccc}
\hline \hline Case & $\eta$ & $\Pi$ & $r_{\text {jet }} / \bar{L}$ & $x_{\mathrm{e}}^{\text {jet }}$ & $x_{\mathrm{H}_{2}}^{\text {jet }}$ & $x_{\mathrm{e}}^{\text {ISM }}$ & $x_{\mathrm{H}_{2}}^{\text {ISM }}$ \\
\hline $\mathcal{A}$ & 10 & 600 & 0.4 & 0.3 & 0. & 0. & 0.40 \\
$\mathcal{B}$ & 1 & 60 & 0.4 & 0.3 & 0. & 0. & 0.40 \\
$\mathcal{C}$ & 0.1 & 6 & 0.4 & 0.3 & 0. & 0. & 0.40 \\
$\mathcal{D}$ & 0.4 & 24 & 0.1 & 0.3 & 0. & 0. & 0.25 \\
$\mathcal{E}$ & 0.4 & 24 & 0.1 & 0.3 & 0. & 0. & 0.25 \\
\hline
\end{tabular}

$\bar{p}=1.381 \times 10^{-10} \mathrm{erg} \mathrm{cm}^{-3}$ (corresponding to a temperature of $100 \mathrm{~K}$ for a purely atomic hydrogen gas at density $\bar{\rho}$ ). Here we will always assume $\Pi=60 \eta$, to preserve the expected high temperature ratio between jet and ISM, with the condition $\Pi>1$. The choice of $\Pi$ (or $\eta$ ) plays a key role, since the pressure ratio affects dramatically the jet behavior and, in particular, the formation of IOS. This, in turn, leads to quite different knot patterns, as we show in the next subsection. Another parameter affecting the structure of IOS, in particular their dimension and spacing, is obviously $r_{\text {jet }}$. Note that cases $\mathcal{A}, \mathcal{B}$, and $C$ differ only for the density ratio $\eta$ (and thus also $\Pi$ ), which is decreased from 10 to 0.1 (thus we move from heavy to light jets). Run $\mathcal{D}$ is a light jet case where parameters have been further optimized to provide knots with a structure similar to that observed, whereas run $\mathcal{E}$ retains the same parameters as in run $\mathcal{D}$, but the ejection is turned off for a while in order to reproduce some observations where two separate chains of knots are found (case of restarting jet, see Sect. 3.4).

\subsection{Formation mechanism of compression regions in under-expanded jets}

We now investigate the formation mechanism of compression regions and IOS in under-expanded jets, that is when $P_{\text {jet }}>$ $P_{\text {ISM }} \Rightarrow \Pi>1$. Under these conditions, just after the nozzle the gas starts to expand downward until the pressure falls below the surrounding local pressure in the cocoon environment. Outward from this pressure equilibrium point the flow is deviated by the inward radial pressure gradient, forming blobs of compressed gas aligned along the axis, which will drive the formation of radiatively emitting knots. This is the formation mechanism of IOS in jets, which is well known in gas dynamics. Here we study how the resulting expansion and compression features depend on the initial parameters.

To this aim the code has been adapted to simulate the $2 \mathrm{D}$ expansion of a circular surface at constant $\xi$, under-expanded with respect to the environment, i.e. to the ISM. The circular surface mimics an expanding slice of gas perpendicular to the jet axis and co-moving with the jet itself. At time $t=0$ it represents the jet nozzle, which has been initialized with a typical set of inflow parameters. In this qualitative model the effects of the longitudinal propagation of the jet, including re-circulating flows, cocoon temporal variability and Kelvin-Helmoltz instabilities arising from the longitudinal velocity shear, have been neglected. In the present section, both adiabatic $(\mathrm{A} 1, \mathrm{~B} 1, \mathrm{C} 1)$ and radiative (A2, B2, C2) simulations are performed, with density ratio $\eta=10,1,0.1$ and pressure ratio $\Pi=600,60,6$, respectively. The other parameters are the same as in the runs $\mathcal{A}, \mathcal{B}, \mathcal{C}$ listed in Table 1, respectively, except for the nozzle size, whose radius is ten times smaller, $0.4 \times 10^{14} \mathrm{~cm}$, also leading to a smaller time evolution scale. In the jet simulations presented in the following

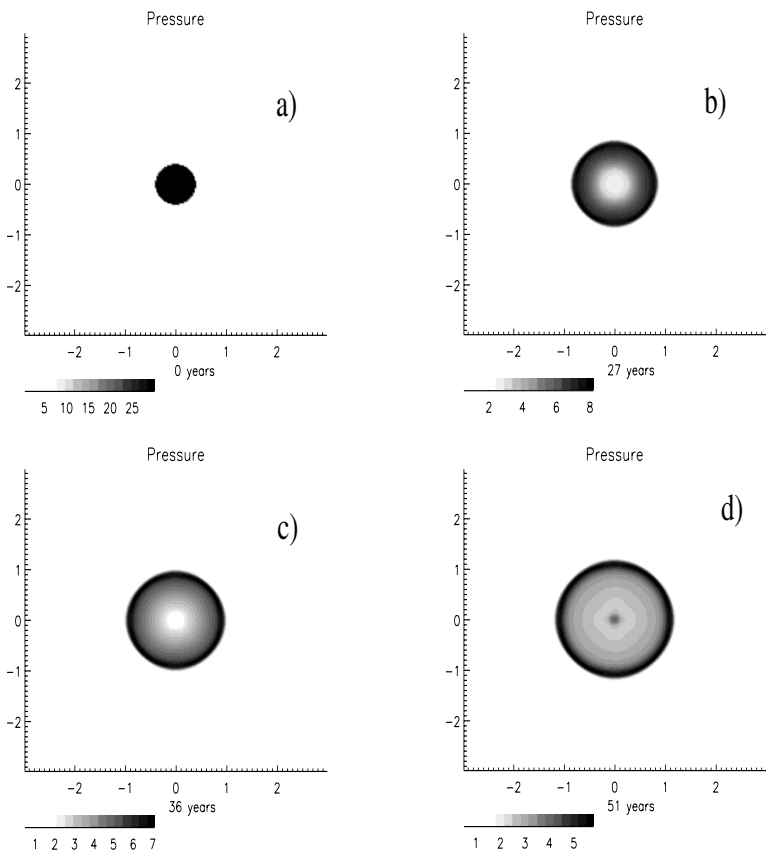

Fig. 1. Case B1. Time evolution of the pressure field. Times are $t=0 \mathrm{yr}$ a), $t=27 \mathrm{yr} \mathrm{b}), t=36 \mathrm{yr} \mathbf{c}), t=51 \mathrm{yr} \mathbf{d})$. The pressure scale is $\bar{p}=1.381 \times 10^{-10} \mathrm{erg} \mathrm{cm}^{3}$, while here the length scale is $10^{14} \mathrm{~cm}$. The lower (white) limit in the color bar is 0.5 , corresponding to the value assumed for the external ISM pressure.

sections a more realistic beam width has been used, to match the estimated mass loss rate.

Case B1 ( $\eta=1, \Pi=60$, adiabatic) is chosen to illustrate the general features of the gas radial evolution as a function of time, described in Fig. 1. Plot (a) represents the initial condition, with uniform pressure distribution inside the jet beam. At $t=27 \mathrm{yr}$ (b) the shell is expanding: a strong pressure wave moves outward, and an evacuated region forms, inside the beam, driven by the internal rarefaction wave. When the inward pressure gradient becomes strong enough, particles from the intermediate shells start to move toward the center (c, $t=36 \mathrm{yr}$ ). This reverse flow can be seen in the velocity pattern of Fig. 2. Particles moving toward the axis finally generate a central peak of pressure, which will result in a radiatively emitting knot (Fig. 1d).

The combined effects of radiative losses and different pressure ratios are shown in Fig. 3, which shows the pressure taken on the axis versus time for all cases. Notice that the pressure jump, coinciding with the formation of the central peak, does not occur at the same time for all runs. In general, the smaller the pressure ratio $\Pi$, the smaller the knot formation time scale, and in turn the smaller the distance from the injection point. In the simulation with $\Pi=6$, case $C$, a knot quite close to the source is produced (at $t=30 \mathrm{yr}$, corresponding to a distance $L \approx 2 \times 10^{16} \mathrm{~cm}$ if the particles co-moved with the jet at the constant velocity of $200 \mathrm{~km} \mathrm{~s}^{-1}$ ). The peak formation is most apparent for case B, where we clearly see a pressure jump, whereas in run $A$, with the highest pressure ratio $\Pi=600$, no knot is generated over the length scale corresponding to $100 \mathrm{yr}$, at the same jet velocity. As far as radiative losses are concerned, these in general depend on the matter density and tend to delay the pressure jump and lower the peak value, as is most apparent by comparing cases B1 and B2. In the radiative case A2, in particular, the jet matter is so overpressured and dense that radiative losses initially result in a quick pressure drop larger than for the adiabatic 


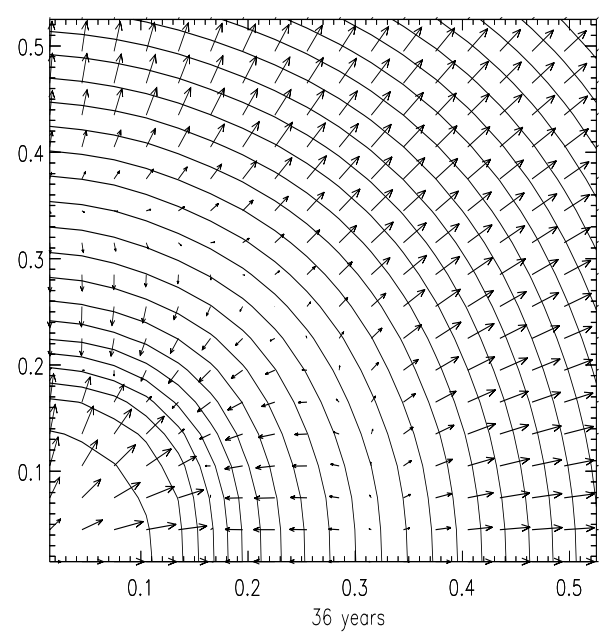

Fig. 2. Case B1. Velocity vectors at $t=36 \mathrm{yr}$. A shell of gas is moving toward the axis pushed by the inward pressure gradient, while the external shells are still expanding outward.

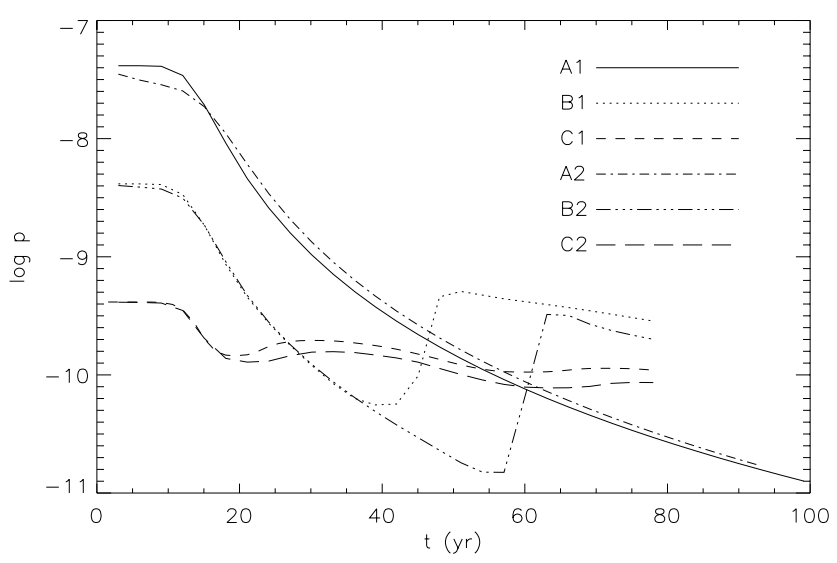

Fig. 3. Plots of the central pressure (in logarithmic scale) versus time for the three values of the pressure ratio $\Pi=600,60,6$ chosen for our simulations (cases A, B, C, respectively). Both adiabatic (1) and radiative (2) runs are shown.

case A1. This radiative loss is also able to keep the pressure value at the center below the adiabatic value at later times. All cases, however, show that the value of the pressure jump is not substantially affected by cooling losses. Instead, on-axis peak and minimum pressure values are smaller than for adiabatic simulations. Radiative losses, in fact, effectively lower the gas adiabatic index $\gamma$, and allow for a denser and cooler central region.

As already anticipated, the nozzle radius is another key parameter that regulates the knots' length scale. The smaller the nozzle radius, the shorter the distance traveled by sound waves in the time it takes to go from the axis to the beam surface and back to the axis, where the compression region forms. We have investigated the suitable initial conditions for a jet simulation. To summarize, under-expanded light jets with small jet/ambient pressure ratio (but still greater than 1), and small nozzle size are expected to produce nodular structures more similar to those observed, in terms of spacing of the knots and closeness to the source. Moreover, contrary to common belief, the formation mechanism described in this section allows for proper motion of knots in real jets. Unlike in this simple model, where the external pressure is constant with time, the beam in a stellar jet is in fact embedded in a variable cocoon. At a given point $\xi$ on the longitudinal axis the local pressure ratio $\Pi_{\mathrm{jc}}(\xi)$ between the pressure of the jet and the cocoon embedding the beam (which is not equal to the constant value of $\Pi$ ) actually changes in time, and the knot has to move to adjust the position to match the new value of $\Pi_{\mathrm{jc}}(\xi)$, according to Fig. 3. Namely, $\Pi_{\mathrm{jc}}(\xi)$ is expected to grow with time, since the pressure inside the beam is steadily fed by the nozzle and stays unchanged, while that in the cocoon decays because of both cooling losses and lateral expansion. Therefore, the knot is expected to move downward following the gas stream, at some fraction of the injection speed, as is shown in next section.

\section{Simulation results and emission maps}

In the present section we show the results of the (radiative) simulations for all the cases listed in Table 1, now restoring the full settings for axisymmetric simulations in $(\xi, r)$. The results are illustrated with 2D maps of the total density on a jet meridional section and, in some cases, with derived synthetic emission maps calculated for the collisionally excited lines [S II] $\lambda \lambda 6716$, 6731. These lines are commonly observed in YSO jets and thus the constructed maps represent a good test of the simulation results against the observations. In some cases the integrated emission from a slice perpendicular to the jet axis (of normalized width $1 \mathrm{~cm}$ ) will be shown as a function of the distance from the source, and hereafter will be labeled as $E(\xi)$. The [S II] emission is calculated from the physical parameters determined in the simulation by using a public routine for a 5-level collisionally excited atom (A. Raga, priv. comm.), assuming that all $\mathrm{S}$ atoms are ionized once and adopting a (constant) relative abundance $\mathrm{S} / \mathrm{H}=1.6 \times 10^{-5}$ (for details see Bacciotti et al. 1995).

\subsection{Heavy and density-matched jets (cases $\mathcal{A}, \mathcal{B}$ )}

Case $\mathcal{A}$, the starting model in the parameter space of Table 1 , represents a heavy $(\eta=10)$, strongly under-expanded jet with pressure ratio $\Pi=600$. This test case does not produce emitting knots. The density field (Fig. 4, top panel) does not reveal any kind of internal structure. Note that in order to allow for a representation of some details of the jet beam, density and emissivity maps in the paper have an aspect ratio of the axes' scales far from unity. In the bottom panel we report the integrated emission function $E(\xi)$. The figure only shows random discontinuities that arise from rings of dense matter in the external cocoon region, rather than from blobs of compressed gas on the axis. Due to the high pressure ratio at the nozzle, the gas expands from the origin to $\approx 150 \bar{L}$, where recompression occurs due to the termination shock corresponding to the Mach disk. Velocity, density and pressure are rather smooth and uniform from the source to the Mach disk (located at $\approx 200 \bar{L}$ at $t=1400 \mathrm{yr}$ ) and comparisons with observed images of $\mathrm{HH}$ objects cannot be attempted. Downstream of the Mach disk a secondary jet forms, accelerated by the local De Laval nozzle generated by a toroidal ring of dense matter that forms around the Mach disk triple point (e.g. Blondin et al. 1990). Such an effect is frequently observed in axisymmetric HD and MHD simulations (e.g. Clarke et al. 1986), while it does not appear in 3D simulations.

Case $\mathcal{B}$, the density-matched run with $\eta=1$ and $\Pi=60$, is still far from showing satisfactory features. Density maps are similar to those of case $\mathcal{A}$, as shown in Fig. 5. Although the jet is more collimated than in case $\mathcal{A}$, no nodular structure is visible, since the pressure ratio $\Pi$ at the nozzle is still too large 

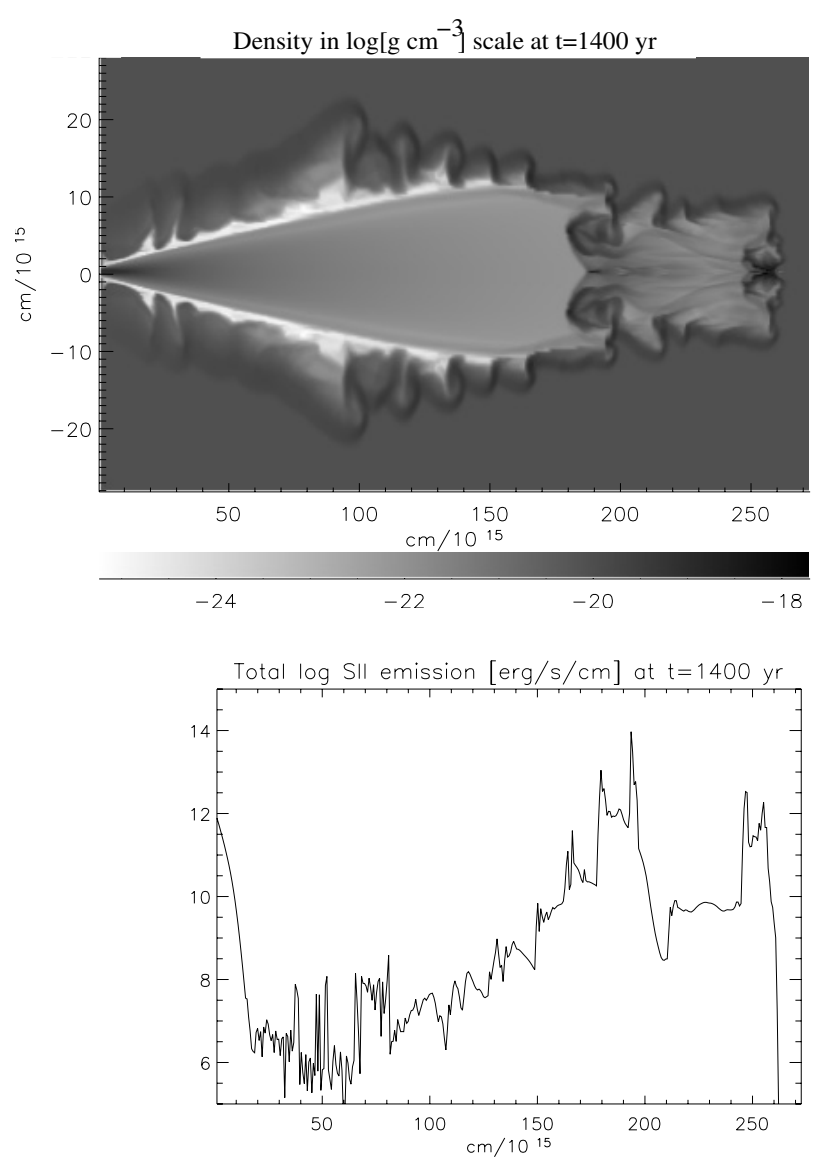

Fig. 4. Case $\mathcal{A}$. Top: density map in logarithmic scale. Bottom: [S II] emission $E(\xi)$, in logarithmic scale, integrated on a cylindrical slice (of thickness $1 \mathrm{~cm}$ ), vs. the distance from the source in units of $\bar{L}$.

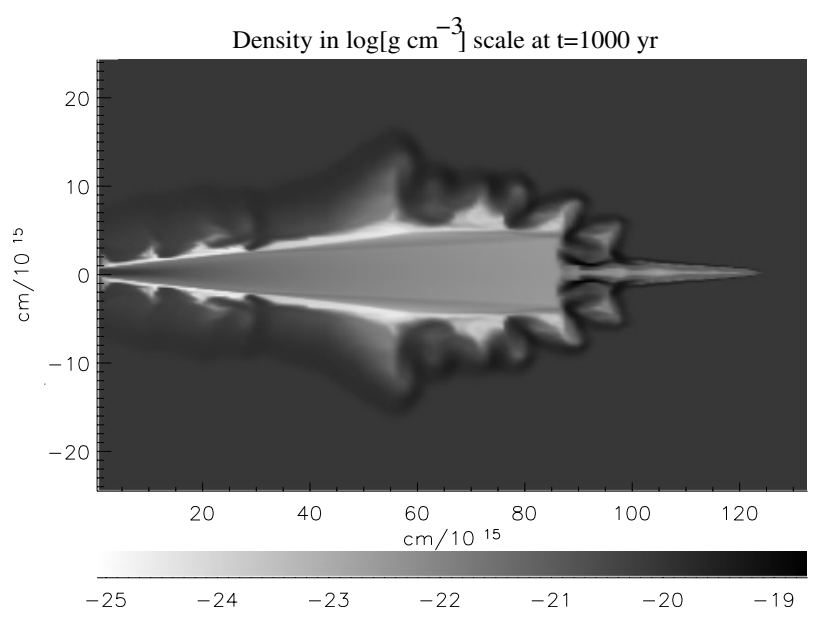

Fig. 5. Case $\mathcal{B}$. Density map in logarithmic scale.

to produce knots over the jet length. Note also that in this case the jet presents a narrower nose cone ahead of the Mach disk.

\subsection{Light jets (case $C$ )}

In this case we have $\eta=0.1$ and $\Pi=6$, all other parameters being unchanged with respect to cases $\mathcal{A}$ and $\mathcal{B}$. The mass loss rate is of the order of $10^{-8} M_{\odot} \mathrm{yr}^{-1}$, in agreement with the estimates derived from observations (Bacciotti et al. 2002). Figure 6 shows the density map (left panel) and a simulated image in the light of [S II] lines (right panel), on the same scale and for the same time $t=1500$ yr. A well defined knot appears at $\xi \approx 90 \bar{L}$ from the source (labeled with A in the synthetic emission map), corresponding to the dark throat observed in the density map. The estimated luminosity of this single knot is $\approx 10^{25} \mathrm{erg} \mathrm{s}^{-1}$. This case confirms that IOS and emission knots appear for small $\Pi$ values. The simple model that has been used to show how blobs of compressed gas form in under-expanded jets, can now be checked. Figure 7 contains a sequence of plots showing the radial profiles $p(r)$ of the pressure, taken at different distances $\xi$ along the jet axis. In each plot the solid line refers to time $t=900 \mathrm{yr}$, while the dotted line refers to the same output time in Fig. 6, $t=1500 \mathrm{yr}$.

We will now discuss the figures in some detail. Panel (a) of Fig. 7 refers to a region quite close to the source, $\xi=0.35 \bar{L}$. The pressure on the axis corresponds to the inflow value that fills the nozzle homogeneously, the external flat profile matches the ISM pressure. The two plots show that close to the source the pattern does not change in time significantly. Panel (b) gives the situation approximately 20 nozzle radii downstream of the source. As particles flow down the nozzle, the beam expands laterally, following the outgoing pressure wave (see Fig. 1b for a comparison with the slice model). Comparison between solid and dotted line in panel (b) shows that the beam undergoes a lateral expansion in time. At $t=1500 \mathrm{yr}$ the beam is wider and the average cocoon pressure is lower with respect to $t=900 \mathrm{yr}$ (the cocoon is defined here as the region bounded by the peak of outgoing pressure wave). Figure $7 \mathrm{c}$, to be compared to Fig. 1c, shows the growth of the inward pressure wave that pushes the gas toward the axis. This inward flow is plotted in Fig. 8, which shows the radial profile of the radial velocity $V_{r}(r)$, taken at the age of $t=900 \mathrm{yr}$ and for $\xi=11 \bar{L}$ (quite near the source, solid line) and for $\xi=21 \bar{L}$, (closer to knot A, dotted line). The former is overall positive (expansion phase), the latter shows that internal rings of matter are moving to the axis (compare also with Fig. 2). In panel (d) the internal compression wave is still moving inward, until it reaches the axis (e, see also Fig. 1d). The peak of pressure on the axis in panel (e) corresponds to knot A in the young jet (i.e. that at $t=900 \mathrm{yr}$ ). The old jet wave is still on the way and reaches the axis in panel (f), approximatively 40 nozzle radii downstream. This spatial delay confirms that as time goes by the knot forms farther and farther away from the source, because of the lower external pressure field, according to Fig. 3. The spatial gap between the peaks in panels (e) and (f) divided by the time interval between what we have labeled as old and young jets measures the knot proper motion. In this case the resulting velocity is too small when compared to observations $\left(V_{\mathrm{knot}} \approx 9 \mathrm{~km} \mathrm{~s}^{-1}\right)$.

\subsection{Light jets with smaller radius (case $\mathcal{D})$}

To allow for the formation of knots over the jet length scale and to reduce the intra-knot spacing, in case $\mathcal{D}$ the nozzle radius $r_{\text {jet }}$ has been reduced to $0.1 \bar{L}$, while $\eta$ has been increased to 0.4 , preserving the usual inflow speed. The increase in density is needed to keep a realistic mass injection rate, by (partially) compensating the loss of area. A spectacular chain of knots appears, very apparent in the $[\mathrm{S} \mathrm{II}]$ emissivity maps of Fig. 9 (left panels), at different output times of jet evolution. A secondary recollimated jet appears at $\xi=130 \bar{L}$ (see the frame at $t=460 \mathrm{yr}$ ), revealed by a light halo in the emissivity, corresponding to a high density ring of matter. As already mentioned in Sect. 3.1, these re-collimation effects are not real, but arise from numerical 

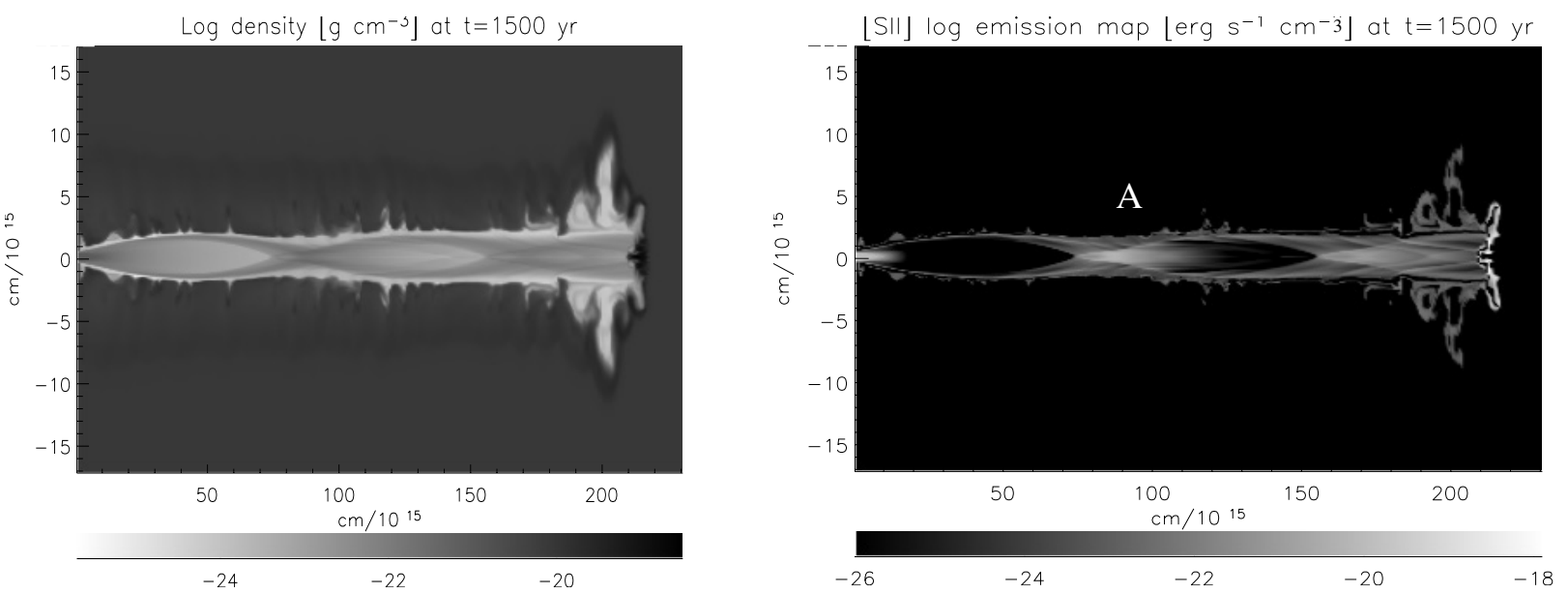

Fig. 6. Case C. Left panel: density map in logarithmic scale. Right panel: [S II] emission map in logarithmic scale. Note the presence of a knot (A). Its estimated luminosity is $\sim 10^{25} \mathrm{erg} \mathrm{s}^{-1}$.
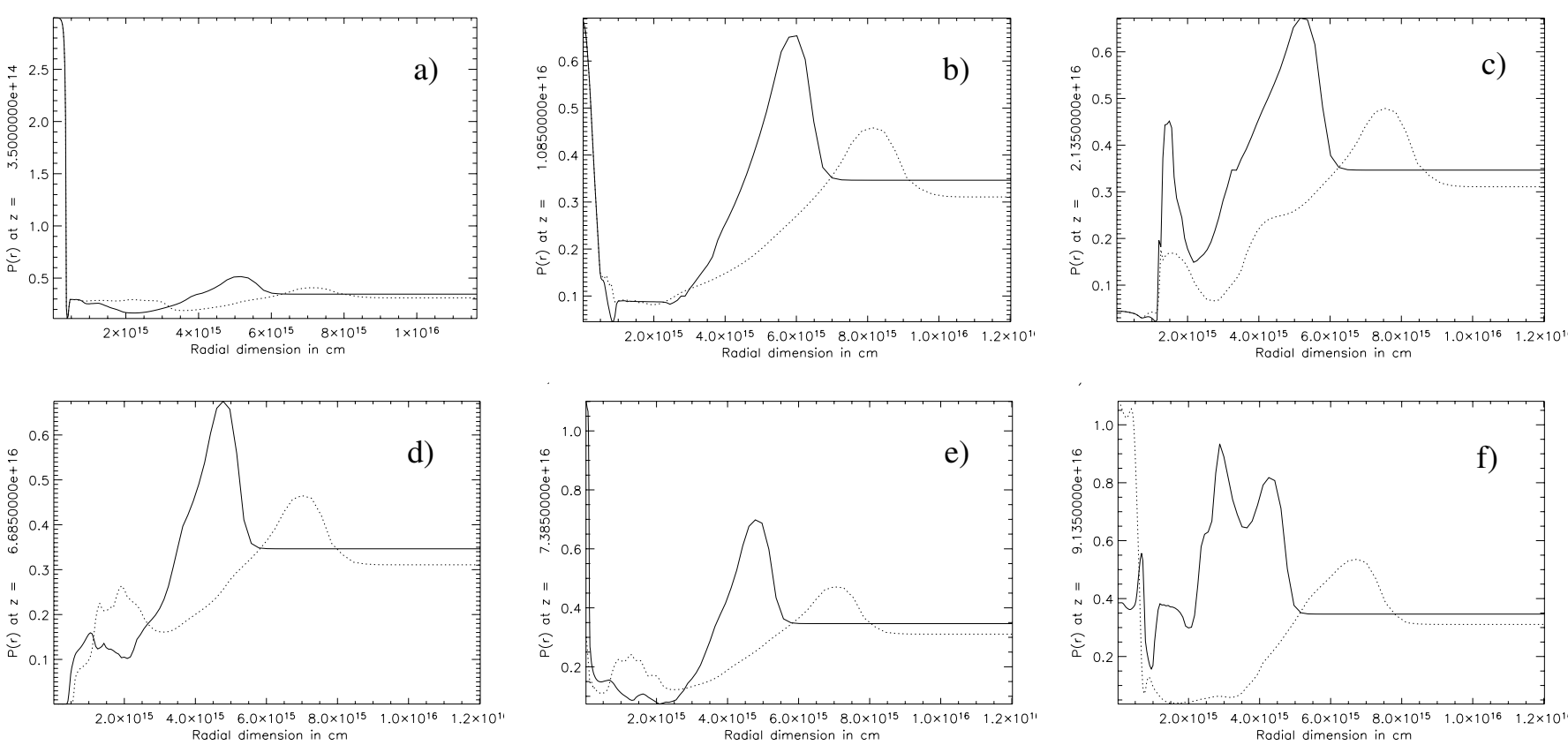

Fig. 7. Case $C$. Pressure $p(r)$ as a function of the radius, at $t=900 \mathrm{yr}$ (solid line) and $t=1500 \mathrm{yr}$ (dotted line). Panels (a)-(f) refer respectively to positions $\xi=0.35 \bar{L}, \xi=11 \bar{L}, \xi=21 \bar{L}, \xi=67 \bar{L}, \xi=74 \bar{L}, \xi=91 \bar{L}$. Pressure and radius scaling changes from picture to picture, to allow for a better representation.

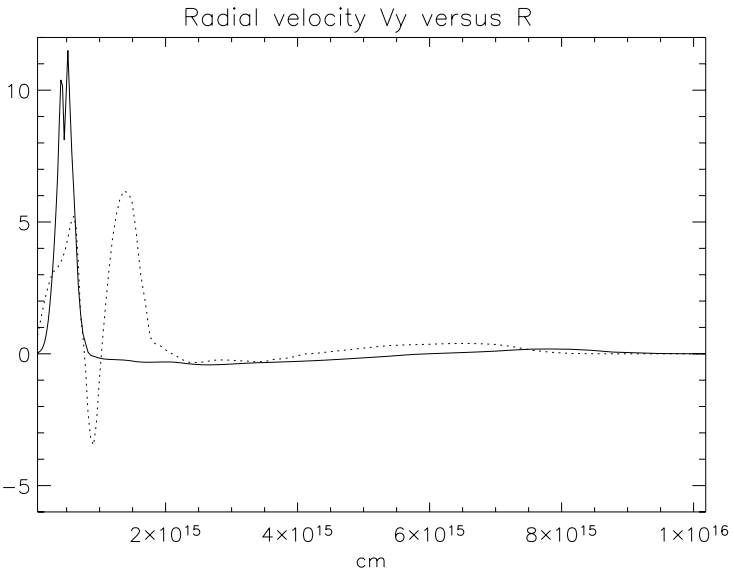

Fig. 8. Case $C$. Radial velocity profile $V_{r}(r)$ at $t=900 \mathrm{yr}, \xi=11 \bar{L}$ (solid line) and $\xi=21 \bar{L}$ (dotted line). effects due to the symmetry of the geometry. Five knots are visible to the left of the re-collimation point (knots A, B, C, D, E), and three to the right (knots F, G, H). Velocities can be estimated by looking at the temporal evolution of the emission function $E(\xi)$, in the same figure (right panels). The position of each knot can be identified by tracking the spatial variations of the corresponding peaks in the 1D plots. Results are shown in Table 2. The estimated velocities are seen to increase with the distance from the nozzle, from $3 \mathrm{~km} \mathrm{~s}^{-1}$ to $64 \mathrm{~km} \mathrm{~s}^{-1}$. Even though these velocities are still far from what observed in real jets (typically knots move at $\approx 70 \%$ of the local flow speed), the trend of increasing velocities with distance from the source is invariably found in our simulations and it is a feature consistent with observations (Eislöffel \& Mundt 1992).

A further comparison with observations of real jets can be attempted by measuring the decay in brightness of the knots over the beam length. If we measure this quantity for the first knots 

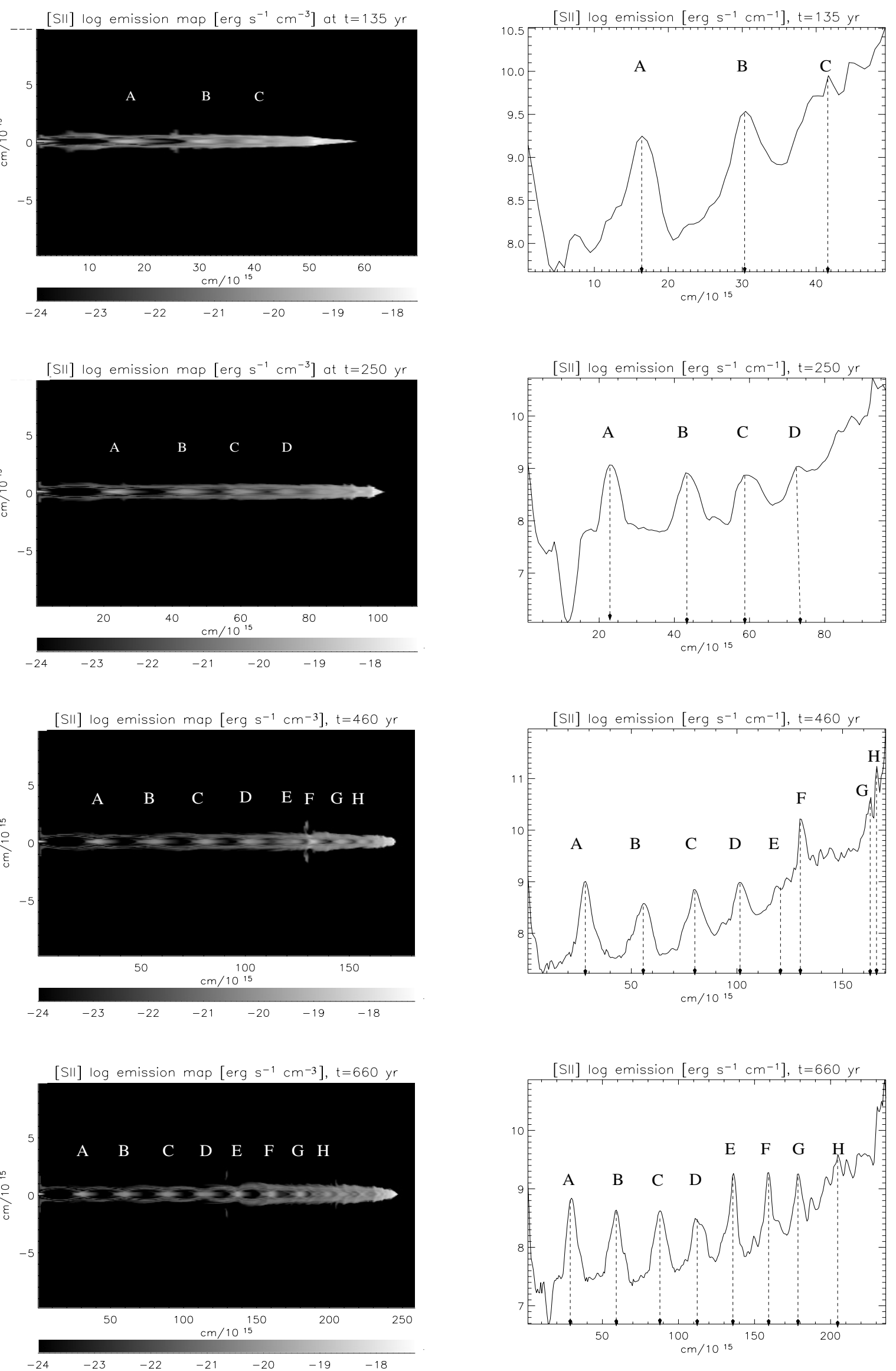

Fig. 9. Case $\mathcal{D}$. [S II] emission map (left panels) and emissivity function $E(\xi)$ (right panels) for different output times. 
Table 2. Case $\mathcal{D}$. Estimated position $\xi$ (in units of $\bar{L}=10^{15} \mathrm{~cm}$ ) and velocity $v$ (in $\mathrm{km} \mathrm{s}^{-1}$ ) along the axis for the knots and output times of Fig. 9.

\begin{tabular}{ccccccccc}
\hline \hline & \multicolumn{3}{c}{$t=135 \mathrm{yr}$} & \multicolumn{3}{c}{$t=250 \mathrm{yr}$} & \multicolumn{3}{c}{$t=460 \mathrm{yr}$} & \multicolumn{2}{c}{$t=660 \mathrm{yr}$} \\
\cline { 2 - 8 } knot & $\xi$ & $v$ & $\xi$ & $v$ & $\xi$ & $v$ & $\xi$ & $v$ \\
\hline A & 16 & - & 23 & 19 & 28 & 7 & 30 & 3 \\
B & 30 & - & 43 & 36 & 55 & 16 & 60 & 8 \\
C & 41 & - & 60 & 52 & 80 & 23 & 90 & 16 \\
D & - & - & 73 & - & 100 & 41 & 112 & 19 \\
E & - & - & - & - & 120 & - & 137 & 27 \\
F & - & - & - & - & 130 & - & 160 & 48 \\
G & - & - & - & - & 162 & - & 180 & 29 \\
H & - & - & - & - & 165 & - & 205 & 64 \\
\hline
\end{tabular}

for the final output time $t=660 \mathrm{yr}$, their brightness decays with an estimated power law exponent $\alpha \approx-0.9$. This value does not match observations and theoretical models, which foresee $\alpha \approx-2$. A value of $\alpha=-1.9$ has been found, as an example, in HH 30 (Ray et al. 1996). In our simulation the value of $\alpha$ could be affected by the beam re-collimation, which changes the brightness slope downward $\xi \simeq 130 \bar{L}$. This is why only the first four knots have been considered in the present estimate. We believe that such a problem will disappear in more realistic 3D simulations.

\subsection{Restarting jets (case $\mathcal{E}$ )}

Case $\mathcal{E}$ retains the same parameters of the previous run. The difference is that a temporal discontinuity has been applied on the initial conditions at the nozzle. Such time-varying initial conditions have nothing to do with the pulsating inflow conditions often invoked in the literature to generate moving knots. In case $\mathcal{E}$ one makes the hypothesis that some discontinuity occurs at the jet source, namely that the flow at the nozzle is switched-off and that, after some time, it is switched-on again, so that former initial conditions are restored. In this way a new, restarting jet forms, which bores its way in the wake of the old one, producing new, interesting features. The motivation for these inflow conditions is provided by the observed formation of leading bow-shocks with temporal spacings of a few hundred years. In the present simulation a temporal variation has been set at the source of the jet with a time frequency small enough with respect to the intra-knot frequency, that one can still consider the overall structure as a steady jet. Note that this kind of variability is not meant to generate high-frequency internal working surfaces as in the pulsating inflow models, since in our simulations knots form because of IOS along the jet beam.

The results of the simulation for case $\mathcal{E}$ are displayed in Fig. 10, where [S II] 2D maps and $E(\xi)$ plots are shown at different output times of the jet evolution. At $t=620 \mathrm{yr}$ the source of the jet has been thus switched off. Both the inflow pressure and density, or mass injection rate, have been reduced by a factor of 10. At $t=720 \mathrm{yr}$ the original initial conditions have been restored, and a new jet forms at the source and starts to propagate in the wake of the old one. In the displayed plots we report emission maps (left panels) and $E(\xi)$ functions (right panels), each row corresponding to different times of the evolution of this two-jet system, from $t=935$ to $t=1050 \mathrm{yr}$. This choice of output times allows us to study in detail the new jet, which exhibits a chain of knots quite close to the source, a typical feature observed in many YSOs (e.g. Reipurth \& Bally 2001).

Interesting results are found by estimating the velocity of such knots (labeled A, B, C, D, E in Fig. 10) from the
Table 3. Case $\mathcal{E}$. Estimated position $\xi$ (in units of $\bar{L}=10^{15} \mathrm{~cm}$ ) and velocity $v$ (in $\mathrm{km} \mathrm{s}^{-1}$ ) along the axis for the knots and output times of Fig. 10.

\begin{tabular}{ccccccccc}
\hline \hline & \multicolumn{2}{c}{$t=935 \mathrm{yr}$} & \multicolumn{2}{c}{$t=970 \mathrm{yr}$} & \multicolumn{2}{c}{$t=990 \mathrm{yr}$} & \multicolumn{2}{c}{$t=1050 \mathrm{yr}$} \\
\cline { 2 - 9 } Knot & $\xi$ & $v$ & $\xi$ & $v$ & $\xi$ & $v$ & $\xi$ & $v$ \\
\hline A & 20 & - & 22 & 18 & 22 & 0 & 25 & 16 \\
B & 36 & - & 40 & 36 & 41 & 16 & 44 & 16 \\
C & 48 & - & 55 & 64 & 60 & 80 & 67 & 37 \\
D & - & - & - & - & 70 & - & 83 & 70 \\
E & - & - & - & - & - & - & 95 & - \\
\hline
\end{tabular}

positions of the corresponding peaks in the integrated emission $E(\xi)$, as done in the previous sub-section. The kinematics of the new jet differs from the old one in two main aspects. The bow-shock propagates at a substantial fraction of the injection velocity $V_{\text {jet }}=200 \mathrm{~km} \mathrm{~s}^{-1}$, while the external bow shock typically propagates at a value which is about half of that speed. Moreover, the knots in the new jet are seen to move with rather high individual velocities, as reported in Table 3. This different behavior with respect to $\mathcal{D}$ is due to the fact that the new jet travels in the low density wake of the old one, rather than in the higher density ambient of the unperturbed ISM. The most remarkable results of this particular experiment are then the morphology and kinematics of the new jet knots, which appear close to the source and with relatively high-speed motions.

\section{Conclusions}

Our simulations show that under-expanded, light jets can naturally generate a pattern of emitting knots that possess proper motions, without invoking temporal variation of the source. In our scenario, knots are due to IOS, which are formed because of standard gas dynamical re-collimation processes, and their proper motion is due to the interaction with a highly timedependent environment, namely the cocoon formed by the propagation of the jet head. The resulting knots are seen to survive radiative cooling and the synthetic images we obtained resemble qualitatively the observations of many $\mathrm{HH}$ objects: the individual velocities are seen to increase with distance from the source and the knots' brightness, on the other hand, is found to decay over the beam length. However, the detailed properties of such knots, in terms of brightness, position in space, proper motion, and intra-knot spacing, obviously heavily depend on initial conditions. Exploring all, or even a large part, of parameter space is well beyond the scope and the possibility of this work, though some final considerations can be made:

- the fact that steady inflow conditions can drive the formation of propagating emitting knots is a remarkable result in itself, since it has often been argued that steady jets could only form steady internal structures;

- intra-knot spacing mostly depends on both $\Pi$, the pressure ratio and $r_{\text {jet }}$, the nozzle radius. The correct choice of these parameters puts the knots length scale and the jet momentum loss in a realistic range;

- the velocities of the knots can reach a significant fraction of the jet bow-shock propagation speed, which, in turn, is typically half of the velocity along the beam (basically the injection velocity). In restarting jets, simulating lowfrequency (compared to the intra-knot frequency) variations of the inflow conditions, both the secondary bow-shock and the newly born knots are seen to propagate faster, reaching up to $40 \%$ of the local flow speed, which is not too far from 

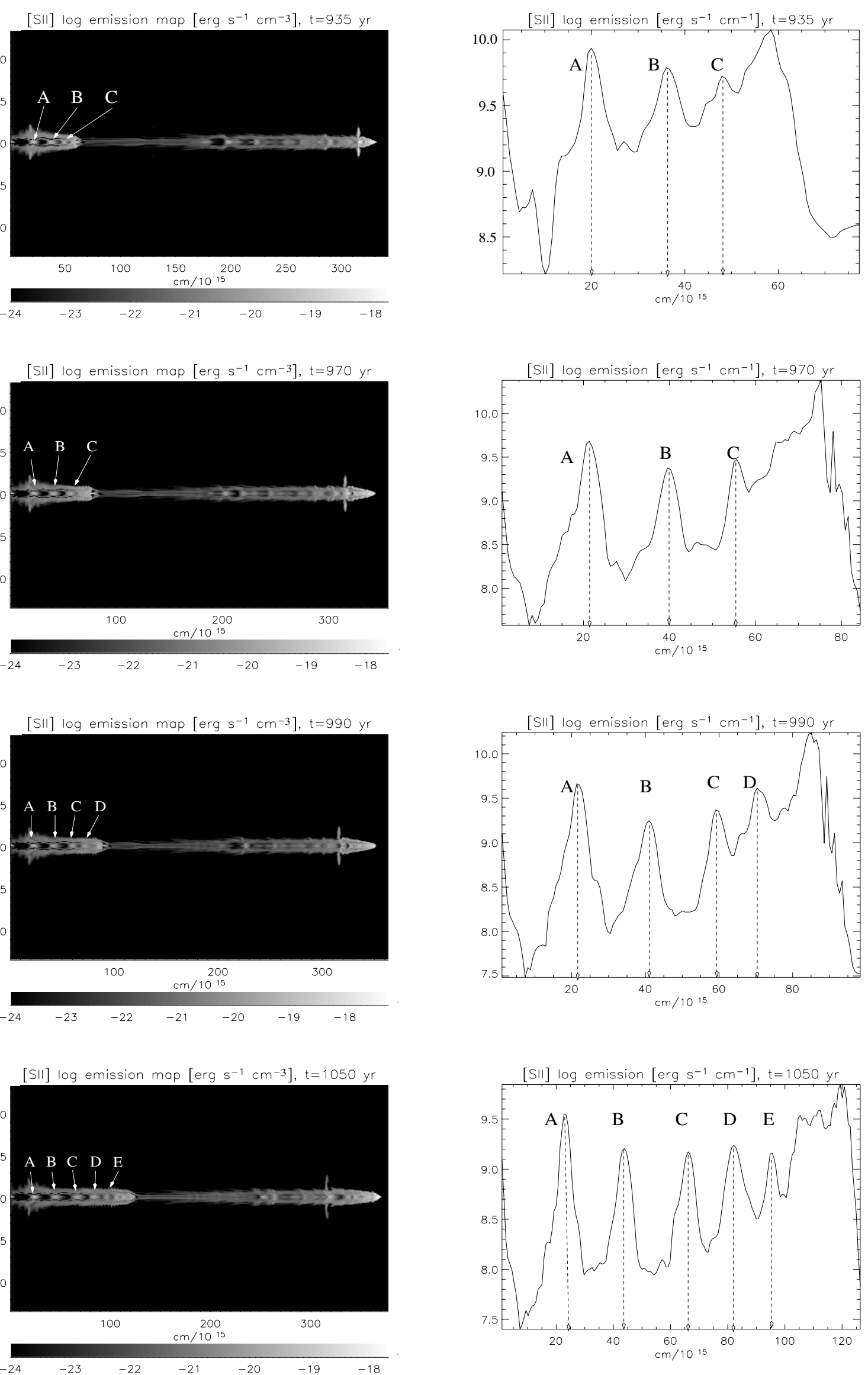

Fig. 10. Case $\mathcal{E}$. [S II] emission map and emissivity function $E(\xi)$ at different output times. 
what is observed in real jets (about 70\%). This promising branch of numerical experiments has just been opened and will be more exhaustively explored in the future;

- the need for 3D calculations arises from some significant discrepancies between numerical results and obervationally determined properties, such as the emissivity decaying exponent with the distance from the source. We claim here that such differences could arise from artificial re-collimation effects due to the assumed hypothesis of axisymmetry, but 3D simulations are needed to prove this statement.

In conclusion, our results show that IOS provide a natural, efficient mechanism for the formation of radiatively emitting knots which possess most of the observed features, such as proper motions with increasing velocities along the jet beam. However, claiming that they are the only driving mechanism is not realistic, and most probably IOS work in co-operation with other mechanisms. In this framework, knots arising from IOS or from local working surfaces generated by inflow conditions fluctuations could either co-exist or work separately in different objects.

Acknowledgements. This work was supported by the INAF (COFIN projects 2002 and 2004), by the ASCI Flash Center, The University of Chicago, and by the CINECA Supercomputing Center. It was also supported in part by the European Community's Marie Curie Actions - Human Resource and Mobility within the JETSET (Jet Simulations, Experiments and Theory) network under contract MRTN-CT-2004 005592. The authors wish to thank Carlo Giovanardi for his help and for fruitful discussions, Claudio Chiuderi for his useful and friendly suggestions, Simone Landi, Guido Bartoli (Scienza Industria e Tecnologia) for technical help, and, last but not least, an anonymous referee for his comments which helped to improve the manuscript.

\section{References}

Bacciotti, F. 2002, in Rev. Mex. Astron. Astrofis. Conf. Ser., 8, ed. W. J. Henney, W. Steffen, L. Binette, \& A. Raga

Bacciotti, F., \& Eislöffel, J. 1999, A\&A, 342, 717

Bacciotti, F., Chiuderi, C., \& Oliva, E. 1995, A\&A, 296, 185

Bacciotti, F., Eislöffel, J., \& Ray, T. P. 1999, A\&A, 350, 917

Bacciotti, F., Ray, T. P., Mundt, R., Eislöffel, J., \& Solf, J. 2002, ApJ, 576, 222

Bally, J., Heathcote, S., Reipurth, B., et al. 2002, AJ, 123, 2627

Blondin, J. M., Fryxell, B. A., \& Konigl, A. 1990, ApJ, 360, 370

Bodo, G., Massaglia, S., Ferrari, A., \& Trussoni, E. 1994, A\&A, 283, 655

Cerqueira, A. H. \& de Gouveia Dal Pino, E. M. 2004, A\&A, 426, L25

Cerqueira, A. H., de Gouveia dal Pino, E. M., \& Herant, M. 1997, ApJ, 489, L185
Cerqueira, A. H., Velázquez, P. F., Raga, A. C., Vasconcelos, M. J., \& de Colle, F. 2006, A\&A, 448, 231

Clarke, D. A., Norman, M. L., \& Burns, J. O. 1986, ApJ, 311, L63

Coffey, D., Bacciotti, F., Woitas, J., Ray, T. P., \& Eislöffel, J. 2004, ApJ, 604, 758

Coffey, D., Bacciotti, J., Ray, T. P., \& Eislöffel, J. 2007, ApJ, in press

Dalgarno, A. \& McCray, R. A. 1972, ARA\&A, 10, 375

de Colle, F. \& Raga, A. C. 2006, A\&A, 449, 1061

Downes, T. P. \& Ray, T. P. 1998 , A\&A, 331, 1130

Eislöffel, J. \& Mundt, R. 1992, A\&A, 263, 292

Falle, S. A. E. G. \& Raga, A. C. 1995, MNRAS, 272, 785

Hartigan, P., Edwards, S., \& Pierson, R. 2004, ApJ, 609, 261

Hartigan, P., Morse, J. A., Reipurth, B., Heathcote, S., \& Bally, J. 2001, ApJ, $559, \mathrm{~L} 157$

Hartigan, P., Heathcote, S., Morse, J. A., Reipurth, B., \& Bally, J. 2005, AJ, 130, 2197

Heathcote, S., Morse, J. A., Hartigan, P., et al. 1996, AJ, 112, 1141

Hollenbach, D. \& McKee, C. F. 1989, ApJ, 342, 306

Lang, K. R. 1975, Astrophysical formulae (Berlin: Springer)

Lavalley-Fouquet, C., Cabrit, S., \& Dougados, C. 2000, A\&A, 356, L41

Lepp, S. \& Shull, J. M. 1983, ApJ, 270, 578

Lorusso, S. 1999, Ph.D. Thesis (Politecnico di Torino)

Mac Low, M.-M. \& Shull, J. M. 1986, ApJ, 302, 585

Martin, P. G., Schwarz, D. H., \& Mandy, M. E. 1996, ApJ, 461, 265

Massaglia, S., Mignone, A., \& Bodo, G. 2005, A\&A, 442, 549

Micono, M., Massaglia, S., Bodo, G., Rossi, P., \& Ferrari, A. 1998, A\&A, 333, 1001

Micono, M., Bodo, G., Massaglia, S., et al. 2000, A\&A, 360, 795

Morse, J. A., Hartigan, P., Cecil, G., Raymond, J. C., \& Heathcote, S. 1992, ApJ, 399,231

O’Sullivan, S. \& Ray, T. P. 2000, A\&A, 363, 355

Ouyed, R. \& Pudritz, R. E. 1997, ApJ, 484, 794

Raga, A. C. \& Kofman, L. 1992, ApJ, 386, 222

Raga, A. C., Canto, J., \& Cabrit, S. 1998, A\&A, 332, 714

Raga, A. C., Velázquez, P. F., Cantó, J., \& Masciadri, E. 2002, A\&A, 395, 647

Raga, A. C., De Colle, F., Kajdič, P., Esquivel, A., \& Cantó, J. 2007, A\&A, in press

Ray, T. P., Mundt, R., Dyson, J. E., Falle, S. A. E. G., \& Raga, A. C. 1996, ApJ, 468, L103+

Reipurth, B. \& Bally, J. 2001, ARA\&A, 39, 403

Reipurth, B., Hartigan, P., Heathcote, S., Morse, J. A., \& Bally, J. 1997, AJ, 114, 757

Reipurth, B., Heathcote, S., Morse, J., Hartigan, P., \& Bally, J. 2002, AJ, 123, 362

Rubini, F., Giovanardi, C., Lorusso, S., Leewin, F., \& Bacciotti, F. 2004, Ap\&SS, 293, 181

Sofia, U. J., Cardelli, J. A., \& Savage, B. D. 1994, ApJ, 430, 650

Spitzer, L. 1978, Physical processes in the interstellar medium (New York: Wiley)

Stone, J. M. \& Hardee, P. E. 2000, ApJ, 540, 192

Stone, J. M. \& Norman, M. L. 1993, ApJ, 413, 210

Suttner, G., Smith, M. D., Yorke, H. W., \& Zinnecker, H. 1997, A\&A, 318, 595

Woitas, J., Bacciotti, F., Ray, T. P., et al. 2005, A\&A, 432, 149 